\title{
COMENTARIO EDITORIAL: Nueva evidencia sobre el rastreo de cáncer prostático: ¿Nuevas recomendaciones?
}

New evidence about prostatic cancer screening: New recommendations?

Agustín Ciapponi*

\begin{abstract}
Resumen
En el contexto de la reciente publicación del ensayo clínico aleatorizado de Goteborg y dos meta-análisis se reanalizó la evidencia sobre el rastreo de cáncer prostático con la determinación de antígeno prostático específico y/o tacto rectal (comparada con la ausencia de rastreo). El autor discute sus resultados, sus limitaciones metodológicas y los metaanaliza junto a los datos que ya existían sobre el tema; arribando a la siguientes conclusiones: la ausencia de efecto del rastreo sobre la mortalidad total y aun tampoco sobre la mortalidad específica por cáncer prostático, sumado a los ya conocidos efectos perjudiciales, impiden avalar la incorporación masiva de la estrategia de rastreo de cáncer prostático. La toma de decisión compartida parece ser la estrategia apropiada a nivel individual.
\end{abstract}

\section{Abstract}

In the context of the recent publication of the Goteborg randomized control trial and two meta-analysis about prostate cancer screening by the determination of prostate specific antigen and/or rectal examination (compared with "absence of screening"); the author discusses their results, their methodological limitations and he meta-analyzes them together with the information that already existed about the topic; arriving at the following conclusion: the lack of effect on total mortality and even not on prostate cancer-specific mortality, coupled with the known adverse effects, prevent endorse the massive incorporation of the strategy of prostate cancer screening. Shared decision making appears to be the appropriate strategy at the individual level.

\footnotetext{
Palabras clave: cáncer prostático, rastreo, mortalidad, diagnóstico. Key words: prostatic cancer, screening, mortality, diagnosis.
}

Ciapponi A. Nueva Evidencia sobre el rastreo de cáncer prostático: ¿Nuevas recomendaciones? Evid. Act. Pract. Ambul. 13(4). 122-124. Oct-Dic. 2010.

\section{Introducción}

Recientemente se publicaron diversos estudios complementando la evidencia existente a la fecha y a la previa editorial publicada en EVIDENCIA durante 2009'.

Además de los dos estudios comentados en este número, el ensayo sueco de Goteborg ${ }^{2}$ y el subsiguiente meta-análisis de Djulbegovic y col. ${ }^{3}$, debe agregarse una actualización 2009 del meta-análisis Cochrane ${ }^{4}$.

El estudio Goteborg ${ }^{2}$ y el subgrupo de 55 a 69 años del estudio ERSPC $^{5}$ fueron los únicos que mostraron beneficios en la mortalidad por cáncer prostático pero ninguno de los seis ensayos publicados a la fecha encontraron beneficios en término de mortalidad global ${ }^{2,5-9}$.
La revisión sistemática Cochrane, que a diferencia de la de Djulbegovic y col. ${ }^{3}$ no incluyó el estudio Goteborg ${ }^{2}$ pero si el sueco Stockholm ${ }^{7}$, meta-analizó un total de cinco ensayos clínicos controlados y aleatorizados (ECCA) con un total de 341.351 participantes y no encontró diferencias estadísticamente significativas en la mortalidad por CP (RR 0,95; IC95\%: 0,85 a 1,07) ni en la mortalidad global (RR 1 IC95\%: 0,98 a 1,02).

El meta-análisis de Djulbegovic ${ }^{3}$ incluyó cinco ECCA que habían evaluado mortalidad y el ERSPC francés ${ }^{10}$, que sólo había evaluado la tasa de diagnóstico de cáncer prostático $(n=387.286)$.

Para integrar toda la información disponible a la fecha, hemos vuelto a meta-analizar los siete ensayos publicados publicados (446.036 participantes totales) cuyas características principales se describen en la tabla 1$)^{2,5}$ a 10 .

Tabla 1: características de los ensayos aleatorizados de rastreo de cáncer prostático.

\begin{tabular}{|c|c|c|c|c|c|c|c|c|c|c|c|}
\hline Estudio & $\begin{array}{c}\text { Grupo } \\
\text { rastreo }\end{array}$ & $\begin{array}{l}\text { Grupo } \\
\text { control }\end{array}$ & $\begin{array}{l}\text { Edad } \\
\text { (años) }\end{array}$ & Pruebas & Aleat. & $\begin{array}{l}\text { Asign. } \\
\text { enmasc. }\end{array}$ & Cegam. & $\begin{array}{l}\text { Seguim. } \\
\text { completo }\end{array}$ & $\begin{array}{l}\text { Sin sesgo } \\
\text { de reporte }\end{array}$ & $\begin{array}{l}\text { Sin otros } \\
\text { sesgos }\end{array}$ & $\begin{array}{l}\text { Seguim. } \\
\text { mediano } \\
\text { (años) }\end{array}$ \\
\hline ERSPC $^{5}$ & 82.816 & 99.184 & $55-74$ & $\mathrm{PSA}+\mathrm{TR}$ & $\mathrm{Si}$ & $?$ & $\mathrm{Si}$ & $\mathrm{Si}$ & $\mathrm{Si}$ & $?$ & 9 \\
\hline ERSPC Francés ${ }^{10}$ & 42.590 & 42.191 & 55 a 69 & PSA & $?$ & $?$ & $?$ & $?$ & $?$ & $?$ & 4 \\
\hline Gothenburg ${ }^{2}$ & 9952 & 9952 & 50 a 64 & PSA & $\mathrm{Si}$ & No & $\mathrm{Si}$ & $\mathrm{Si}$ & $\mathrm{Si}$ & $?$ & 14 \\
\hline Norrkoping ${ }^{8}$ & 1.494 & 7.532 & 50 a 69 & $\begin{array}{c}\text { TR } \\
\text { PSA+TRa }\end{array}$ & No & No & & $?$ & $?$ & $\mathrm{Si}$ & \begin{tabular}{|l|}
13 (diagn.) \\
15 (muerte)
\end{tabular} \\
\hline $\mathrm{PLCO}^{6}$ & 38.343 & 38.350 & 55 a 74 & PSA+TR & $\mathrm{Si}$ & $\mathrm{Si}$ & $\mathrm{Si}$ & No & $\mathrm{Si}$ & $\mathrm{Si}$ & 11.5 \\
\hline Quebec $^{9}$ & 31133 & 15353 & 45 a 80 & $\mathrm{PSA}+\mathrm{TR}$ & $?$ & No & $?$ & & $?$ & No & 11 \\
\hline Stockholm ${ }^{7}$ & 23.74 & 24.772 & 55 a 70 & $\mathrm{PSA}+\mathrm{TR}$ & $?$ & No & $\mathrm{Si}$ & $\mathrm{Si}$ & $?$ & $\mathrm{Si}$ & 15 \\
\hline
\end{tabular}

TR: Tacto rectal; PSA: Antígeno Prostático Específico. a Desde 1993.

En el grupo rastreo se identificaron $37 \%$ más CP y casi el doble de CP estadio I que en el grupo control (tabla 2, figura 1) pero no se encontraron diferencias significativas en la mortalidad global ni en la mortalidad por cáncer prostático (tabla 2, figura 2 y figura 3 ). 
Tabla 2: resumen de la evidencia sobre rastreo de cáncer prostático.

\begin{tabular}{|c|c|c|c|c|c|c|}
\hline & Resultados & $\begin{array}{c}\text { Eventos \%o } \\
\text { control }\end{array}$ & $\begin{array}{l}\text { Eventos \%o } \\
\text { Screening }\end{array}$ & RR (IC95\%) & Población & GRADE* \\
\hline \multirow[t]{2}{*}{ Mortalidad } & Global & 200 & 198 (196 a 202) & $0,99(0,98$ a 1,01$)$ & 305.743 & Moderado \\
\hline & Por cáncer prostático & 8 & 7 (6 a 8) & $0,91(0,81$ a 1,01$)$ & 361.255 & Moderado \\
\hline \multirow{4}{*}{$\begin{array}{c}\text { Diagnóstico de } \\
\text { cáncer prostático }\end{array}$} & Global & 44 & 60 (51 a 71) & $1,37(1,16$ a 1,62$)$ & 399.550 & Moderado \\
\hline & Estadio I & 11 & 21 (13 a 34) & $1,95(1,22$ a 3,13$)$ & 332.743 & Bajo \\
\hline & Estadio II & 23 & 32 (23 a 45) & $1,39(0,99$ a 1,95$)$ & 332.743 & Muy Bajo \\
\hline & Estadios III ylV & 5 & $5(4$ a 5$)$ & $0,94(0,85$ a 1,04$)$ & 332.743 & Moderado \\
\hline
\end{tabular}

Figura 1: diagnóstico de cáncer prostático.

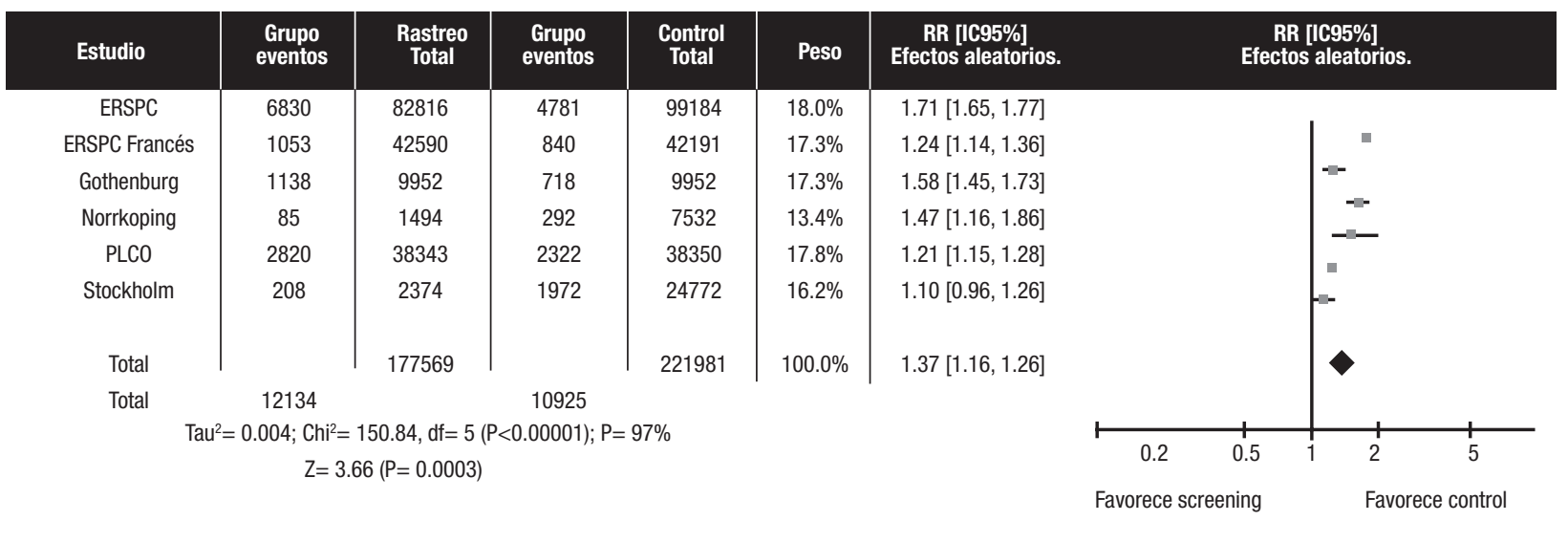

Figura 2: mortalidad global.

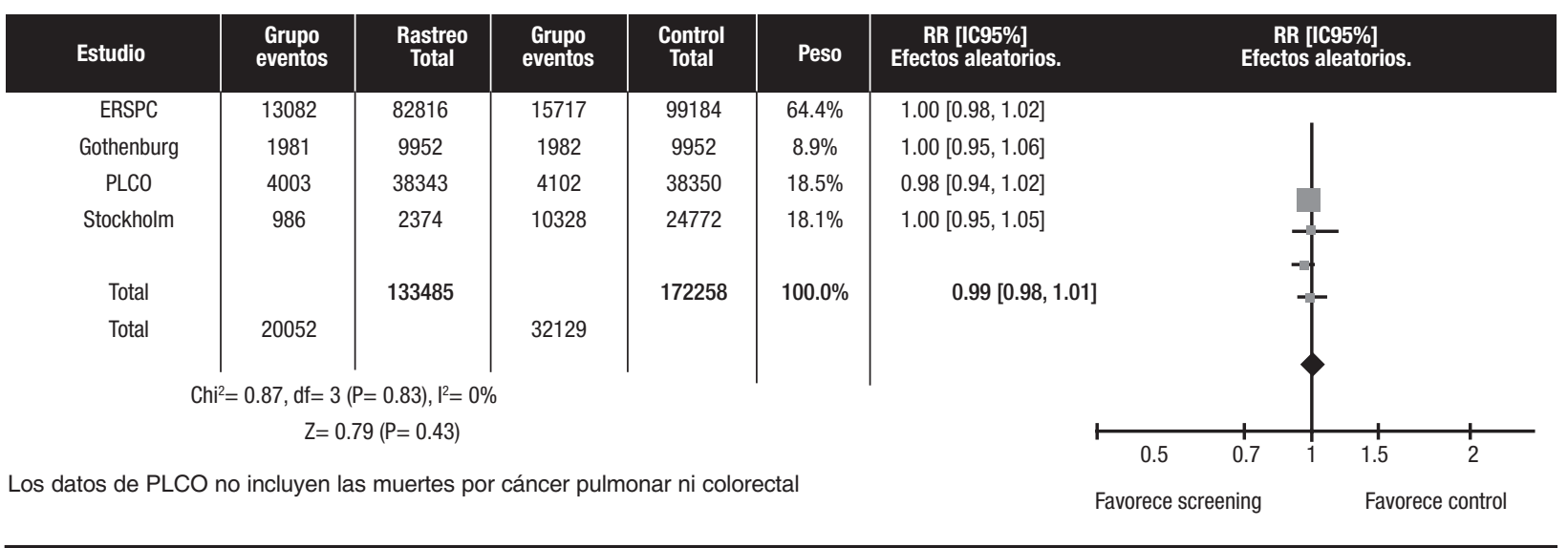

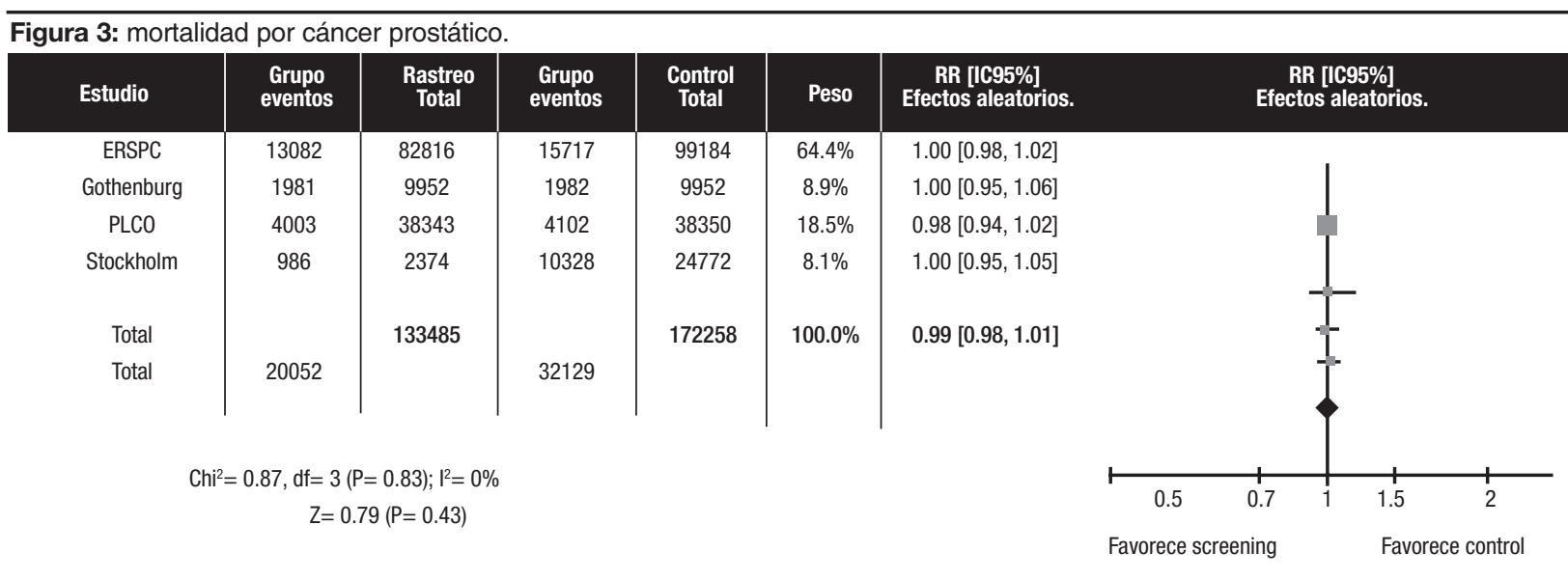


El análisis del subgrupo de los estudios de bajo riesgo de sesgo ${ }^{6} y$ de los de alto riesgo ${ }^{2,7-10}$ no modificó estos resultados. Sólo se encontraron diferencias significativas en el subgrupo que había iniciado su rastreo a los 50 años (RR 0,82; IC95\%: 0,71 a 0,94) pero no si lo había iniciado a los 45 ó a los 55 años.

Aun con la publicación del estudio Goteborg², con un resultado llamativamente más beneficioso que cualquiera de sus predecesores, los resultados sumarios parecen mantener el "status quo" con respecto a las conclusiones y recomendaciones del 2009.

Varios estudios sobre rastreo de CP están en curso y se espera que proporcionen evidencia adicional sobre los beneficios y los daños del rastreo, así como sobre el efecto de distintas opciones terapéuticas para pacientes con resultados positivos.

El ensayo británico Protec ${ }^{11}$ y su extensión CAP, están en marcha pero se esperan resultados finales para 2013 y 2015 , respectivamente. En este ensayo aleatorizado por grupos o "clusters"* (consultorios) cerca de 460.000 hombres de 50 a 69 años fueron asignados a cuidados usuales o a rastreo poblacional con antígeno prostático específico (en inglés con PSA) y con biopsia cuando el PSA supera $3 \mathrm{ng} / \mathrm{mL}$, seguido de la aleatorización de los participantes diagnosticados con cáncer prostático (alrededor de 1500) a cirugía radical, radioterapia tridimensional o vigilancia activa.

En EE.UU., desde 1994 a 2002 el estudio PIVOT asignó al azar 731 hombres a prostatectomía radical o a vigilancia activa ${ }^{12}$. El resultado final se espera para 2011 ó 2012.

Por último, el estudio canadiense START tiene previsto asignar al azar a 2.130 hombres con cáncer próstatico localizado de bajo riesgo a vigilancia activa versus intervenciones tempranas con intención curativa ${ }^{13}$.

\section{Conclusión}

Podemos concluir que en las ramas "rastreo" de los ensayos se detectó un 40\% más de CP que en las ramas "control", pudiendo ser menor esta cifra en la vida real que en la hallada en los estudios experimentales.
Hay suficiente evidencia como para afirmar que el rastreo no mejora la mortalidad global. Con más de 300.000 pacientes reclutados y un estrecho intervalo de confianza en torno del riesgo relativo igual a uno, es improbable que nuevos estudios, aun con gran tamaño muestral modifiquen este robusto resultado.

Sin embargo, continúa habiendo insuficiente evidencia como para apoyar o rechazar el rastreo respecto de su efecto sobre la mortalidad por CP. Aunque las diferencias continúan siendo estadísticamente no significativas no puede descartarse una reducción de la misma. Hasta es probable que se alcance un efecto de alrededor del $10 \%$ de reducción de muerte por CP con la publicación de los nuevos ensayos o la extensión en el seguimiento de los mismos. Vale destacar que este (todavía potencial) beneficio se obtendría pagando el precio, ya confirmado, de resultados falsos positivos, biopsias innecesarias, aumento de la ansiedad y lo que es más grave aún, un substancial sobrediagnóstico (diagnóstico de CP que no tendrán consecuencias en la salud) con las consecuentes intervenciones y frecuentes complicaciones terapéuticas que se evitarían sin el rastreo. Siguiendo con este punto, se estima que el nivel de sobrediagnóstico puede oscilar entre un $25 \%^{2,6}$ y hasta más del $70 \%$ en el estudio ERSPC5.

Queda reforzada la reciente recomendación en contra del rastreo de cáncer prostático a partir de los 75 años. De hecho en el estudio ERSPC ${ }^{5}$, cuando el rastro se iniciaba entre los 69 y 74 años, se apreció una tendencia estadísticamente no significativa, a mayor mortalidad de CP en el grupo rastreo.

El rol del tacto rectal es aun más cuestionable, por sus peores características operativas y porque se desconoce su efecto adicional o en reemplazo del PSA.

Nuestra postura es hacia propiciar la toma de decisión compartida entre el médico y el paciente sobre el rastreo con PSA, facilitada en lo posible por materiales apropiados de educación para el paciente, tal como lo recomiendan la mayoría de las guías.

Referencias

1. Ciapponi A. COMENTARIO EDITORIAL: El rastreo de cáncer de próstata y su eterno dilema: ¿es beneficioso o perjudicial? Evidencia aten primaria 2009;12:42-6.

2. Hugosson $\mathrm{J}$ y col. Mortality results from the Goteborg randomised population-based prostate-cancer screening trial. Lancet Oncol 2010;11:725-32.

3. Djulbegovic M y col. Screening for prostate cancer: systematic review and meta-analysis of randomised controlled trials. BMJ 2010;341:c4543.

4. llic D y col. Screening for prostate cancer. Cochrane Database Syst Rev 2006;3:CD004720.

5. Schroder F y col. Screening and prostate-cancer mortality in a randomized European study. N Engl J Med 2009;360:1320-8.

6. Andriole G y col. Mortality results from a randomized prostate-cancer screening trial. N Engl J Med 2009:360:1310-9.

7. Kjellman A y col. 15-year followup of a population based prostate cancer screening study. J Urol 2009;181:1615-21; discussion 21.

8. Sandblom G y col. Clinical consequences of screening for prostate cancer: 15 years follow-up of a randomised controlled trial in Sweden. Eur Urol 2004;46:717-23; discussion 24.

9. Labrie F y col. Screening decreases prostate cancer mortality: 11-year follow-up of the 1988 Quebec prospective randomized controlled trial. Prostate 2004;59:311-8.

10. Jegu J y col. [Results and participation factors to the European Randomized study of Screening for Prostate Cancer (ERSPC) with Prostate Specific Antigen: French departments of Tarn and Herault]. Prog Urol 2009;19:487-98.

11. Donovan J y col. Prostate Testing for Cancer and Treatment (ProtecT) feasibility study. Health Technol Assess 2003;7:1-88.

12. Wilt T y col. Prostate cancer Intervention Versus Observation Trial:VA/NCI/AHRQ Cooperative Studies Program \#407 (PIVOT): design and baseline results of a randomized controlled trial comparing radical prostatectomy to watchful waiting for men with clinically localized prostate cancer. Contemp Clin Trials 2009;30:81-7.

13. Wilt T. SPCG-4: a needed START to PIVOTal data to promote and protect evidence-based prostate cancer care. J Natl Cancer Inst 2008;100:1123-5. 